\title{
Study of High-dose X-ray Radiation Damage of Silicon Sensors
}

\author{
Robert Klanner ${ }^{\mathrm{a}, *}$, Eckhart Fretwurst ${ }^{\mathrm{a}}$, Ioana Pintilie ${ }^{\mathrm{b}}$, Joern Schwandt ${ }^{\mathrm{a}}$, Jiaguo Zhang ${ }^{\mathrm{a}}$ \\ ${ }^{a}$ University of Hamburg, Hamburg, Germany \\ ${ }^{b}$ National Institute of Materials Physics, Bucharest, Romania
}

\begin{abstract}
The high intensity and high repetition rate of the European X-Ray Free-Electron Laser, presently under construction in Hamburg, will require pixel sensors which can stand X-ray doses up to 1 GGy for 3 years of operation. Within the AGIPD Collaboration the Hamburg group has systematically studied X-ray damage in silicon sensors for the dose range between 10 kGy and 1 GGy using strip sensors and test structures fabricated on high-ohmic $n$-type silicon from four different vendors. The densities of oxide charges, interface traps and surface current as function of dose and annealing conditions have been determined. The results have been implemented in TCAD simulations, and the radiation performance of strip sensors and guard-ring structures has been simulated and compared to experimental results. Finally, with the help of detailed TCAD simulations, the layout and technological parameters of the AGIPD pixel sensor have been optimized. It is found that the optimization for silicon sensors exposed to high X-ray doses is significantly different from that for non-irradiated sensors, and that the specifications of the AGIPD sensor can be met.
\end{abstract}

Keywords: XFEL, silicon pixel sensor, plasma effect, X-ray radiation damage, sensor optimization.

\section{Introduction}

The European X-Ray Free-Electron Laser (EuXFEL) [1, 2], ${ }^{32}$ planned to start operation in 2016, will provide X-ray beams with unique features: A brilliance which is 8 orders of magnitude higher than the most brilliant synchrotron-radiation beams ${ }^{35}$ for wavelengths in the Angström region, full transverse coher- ${ }^{36}$ ence, a pulse length of about $10 \mathrm{fs}$, and pulse trains of 2700 pulses with $220 \mathrm{~ns}$ spacing every $100 \mathrm{~ms}$. These unique fea- ${ }_{37}$ tures pose major challenges for imaging detectors, in particular [3, 4]: A dynamic range of 0,1 to more than $10^{4}$ photons of 38 typically $12.4 \mathrm{keV}$ per pixel, a radiation tolerance for doses up ${ }_{39}$ to 1 GGy for 3 years of operation, a good detection efficiency 40 for X-rays with energies between 3 and $20 \mathrm{keV}$, and minimal 41 inactive regions at the edge of the sensors.

Within the AGIPD (Adaptive Gain Integrating Pixel Detec- 43 tor) Collaboration $[5,6]$ the Hamburg group has studied the 44 consequences of these requirements for $p^{+} n$-silicon sensors and 45 optimized the design of the AGIPD sensor. From the study of 46 the plasma effect, which occurs at high instantaneous X-ray 47 densities [7, 8,9], it has been concluded that, for a sensor of 48 a thickness of $500 \mu \mathrm{m}$, an operating voltage above $500 \mathrm{~V}$ is 49 needed to achieve a sufficiently high electric field to limit the 50 spatial spread of the charge carriers and to achieve a charge- 51 collection time compatible with the $220 \mathrm{~ns}$ spacing of the Eu- 52 XFEL pulses. Studies of the charge collection in segmented ${ }_{53}$ sensors after irradiation with different X-ray doses [10, 11] have 54 shown that, depending on X-ray dose, biasing history and envi- 55 ronmental parameters like relative humidity, losses of holes or 56 electrons occur. However, these effects have little relevance for 57 the EuXFEL applications.

*Corresponding author. Email: robert.klanner@desy.de.
In this paper we summarize the results on the main effects of X-ray radiation damage, in particular the increase of oxidecharge density, the formation of $\mathrm{Si}_{-} \mathrm{SiO}_{2}$-interface traps, their impact on dark current and breakdown voltage, and the optimization of the design of the AGIPD sensor for high operating voltages for X-ray doses between 0 and 1 GGy.

\section{X-ray radiation damage of $p^{+} n$-silicon sensors}

The X-ray energies at the EuXFEL are well below the threshold energy for the formation of defects in the silicon bulk, and only defects in the dielectric, at the $\mathrm{Si}-\mathrm{SiO}_{2}$ interface, and interfaces between dielectrics are generated. The effects of X-ray radiation damage are discussed in detail in [12, 13]. Here we give only a very short summary. In $\mathrm{SiO}_{2} \mathrm{X}$-rays produce on average one $e h$ pair every $18 \mathrm{eV}$ of deposited energy. Depending on ionization density and electric field, a fraction of the $e h$ pairs recombine. The remaining charge carriers move in the $\mathrm{SiO}_{2}$ by diffusion and, if an electric field is present, by drift. Electrons, due to their high mobility and relatively low trapping probability, leave the $\mathrm{SiO}_{2}$. However holes, which move via polaron hopping, are typically captured by deep holes in the $\mathrm{SiO}_{2}$ or at the $\mathrm{Si}-\mathrm{SiO}_{2}$ interface, which results in fixed positive charge states. We denote the density of oxide charges by $N_{o x}$, the surface-current density by $J_{\text {surf }}$, and the density of interface traps as function of their energy $E$ relative to the conduction band by $D_{i t}(E)$ with units $1 /\left(\mathrm{eV} \cdot \mathrm{cm}^{2}\right)$. The interface traps, if exposed to an electric field, act as generation centers and generate a surface current.

For a realistic simulation and optimization of sensors, values of $N_{o x}, N_{i t}$, the effective number of interface traps, and $J_{\text {surf }}$ as function of dose are required. We therefore have ir- 


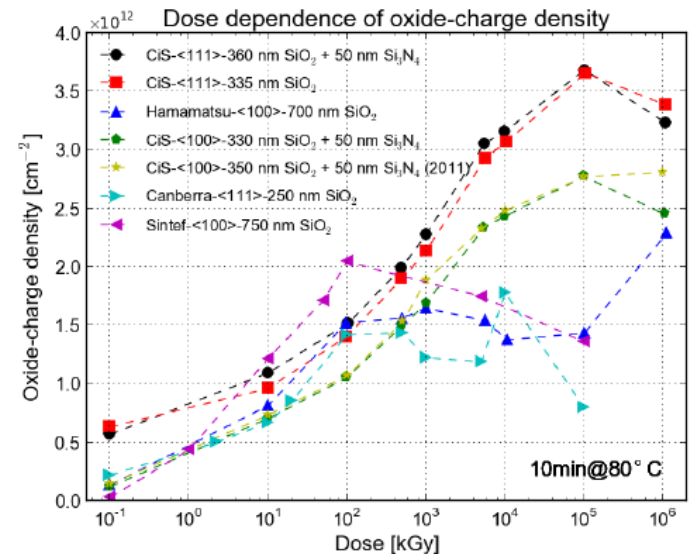

Figure 1: Dependence of the surface-charge density, $N_{o x}$, on X-ray dose ob- ${ }^{118}$ tained from measurements on MOS capacitors from 4 different vendors after annealing for 10 minutes at $80^{\circ} \mathrm{C}$.

In order to determine $N_{0}, \mathrm{C} / \mathrm{G}-\mathrm{V}$ measurements on the MOS-C for frequencies between 1 and $1000 \mathrm{kHz}$ were made.122 $N_{o x}$, which just shifts the $\mathrm{C} / \mathrm{G}-\mathrm{V}$ curves along the $\mathrm{V}$ axis, has ${ }_{123}$ been derived from the voltage shift of the calculated $1 \mathrm{kHz} C-\mathrm{V}_{124}$ curve with respect to the data. After this shift the calculated ${ }_{125}$ $\mathrm{C} / \mathrm{G}-\mathrm{V}$ curves provide a fair description of the measurements 126 for all frequencies and radiation doses.

Figure 1 shows the results for the thus determined values of ${ }_{128}$ $N_{o x}$. The CMOS-C had been annealed for 10 minutes at $80^{\circ} \mathrm{C}_{129}$ to reach a stable state with respect to short-term annealing. $\mathrm{Up}_{130}$ to an X-ray dose of approximately $100 \mathrm{kGy} N_{o x}$ increases, and ${ }_{131}$ values of about $2 \cdot 10^{12} \mathrm{~cm}^{-2}$ are reached. Above this dose for ${ }_{132}$ some MOS-Cs $N_{o x}$ saturates, for others it continues to increase. ${ }_{133}$ radiated test structures from 4 different vendors (Canberra [14], 99 CiS [15], Hamamatsu [16], Sintef [17]) built on high-ohmic 100 $n$-type silicon $(3-14 \mathrm{k} \Omega \cdot \mathrm{cm})$, with different crystal orien-101 tations $(\langle 111\rangle$ and $\langle 100\rangle)$, and different dielectra $\left(\mathrm{SiO}_{2}\right.$ and ${ }_{102}$ $\left.\mathrm{SiO}_{2}+\mathrm{Si}_{3} \mathrm{~N}_{4}\right)$. The structures used were MOS Capacitors, 103 MOS-C, and Gate-Controlled Diodes [18], GCD. The irra-104 diations were performed at the "white" X-ray beam F4 at ${ }_{105}$ DORIS III, which had a mean energy of $12 \mathrm{keV}$ and dose rates 100 between 1 and $200 \mathrm{kGy} / \mathrm{s}[19,20]$. Irradiations were performed ${ }_{107}$ for dose values between $1 \mathrm{kGy}$ and $1 \mathrm{GGy}$.

In order to determine $D_{i t}(E)$, Thermal Dielectric Relaxation ${ }_{109}$ Current measurements, TDRC, on the MOS-C were made. $\mathrm{In}_{110}$ these measurements the MOS-C, biased in accumulation, was 111 cooled down to a temperature of $10 \mathrm{~K}$ to freeze the electrons $\mathrm{s}_{112}$ in the interface traps. Then the MOS- $\mathrm{C}$ was biased to deep de-113 pletion, heated up with a constant heating rate $\beta=0.183 \mathrm{~K} / \mathrm{s}_{114}$ to $290 \mathrm{~K}$, the current $I_{T D R C}(T)$ due to the release of the trapped ${ }_{115}$ electrons measured, and $D_{i t}(E)$ extracted. To obtain quantita-116 tive results, the $D_{i t}(E)$ spectrum was fitted by 3 Gauss func-117 tions [20]. From measurements with different heating rates $\beta$, the charge-carrier cross sections for the three levels were estimated. Following [21], this information was fed into an equivalent RC-circuit model and the voltage dependence of the capacitance/conductance, $\mathrm{C} / \mathrm{G}-\mathrm{V}$, for different frequencies evaluated, assuming acceptor-like interface traps.
We have verified that the spread in $N_{o x}$ for different MOS-Cs from the same producer is small, so that the large spread is attributed to the different technologies and crystal orientations.

For determining the surface-current densities, I-V measurements on Gate Controlled Diodes, GCD, were performed. The diodes were biased to $-12 \mathrm{~V}$, the voltage on the gate varied from accumulation via depletion to inversion, and the diode current measured. The surface-current density, $J_{\text {surf }}$, was obtained by dividing $I_{\text {surf }}$, the difference in current between depletion and accumulation, by the gate area. For the calculation of $J_{\text {surf }}$ it has been assumed, that the entire gate area is depleted, which may not be correct for all GCDs at high currents. It has been estimated that in this way the value of $J_{\text {surf }}$ could be underestimated by at most $50 \%$. Figure 2 shows the results for $J_{\text {surf }}$. As for $N_{o x}$, the values of $J_{\text {surf }}$ saturate at dose values between 1 and $10 \mathrm{MGy}$. The maximal values of $J_{\text {surf }}$ vary between 1.5 and $6.5 \mu \mathrm{A} / \mathrm{cm}^{2}$, which we again attribute to differences in technology. At higher doses $J_{\text {surf }}$ decreases, which is not yet understood.

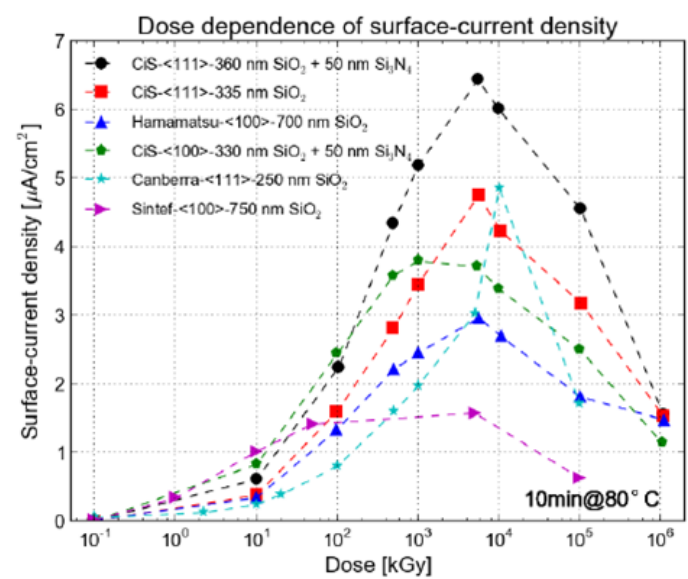

Figure 2: Dependence of the surface-current density $J_{\text {surf }}$ on X-ray dose obtained from measurements on Gate Controlled Diodes from different vendors after annealing for 10 minutes at $80^{\circ} \mathrm{C}$.

\section{Sensor optimization}

The results of the studies of the plasma effect have shown that for experiments with high instantaneous $\mathrm{X}$-ray intensities, e.g. $10^{4} \mathrm{X}$-ray photons per pulse in a $200 \mu \mathrm{m} \times 200 \mu \mathrm{m}$ pixel, operating voltages well above $500 \mathrm{~V}$ are required for $500 \mu \mathrm{m}$ thick sensors. The problem of reaching a high breakdown voltage for high radiation-induced oxide-charge densities is illustrated in Figure 3, which shows the electric field close to the $\mathrm{Si}-\mathrm{SiO}_{2}$ interface from a 2D TCAD simulation of a $p^{+} n$-strip sensor biased at $500 \mathrm{~V}$ for two values of $N_{o x}$. Whereas the maximal electric field $10 \mathrm{~nm}$ below the $\mathrm{Si}-\mathrm{SiO}_{2}$ interface is $50 \mathrm{kV} / \mathrm{cm}$ for $N_{o x}=10^{11} \mathrm{~cm}^{-2}$, it is $450 \mathrm{kV} / \mathrm{cm}$ for $N_{o x}=2 \cdot 10^{12} \mathrm{~cm}^{-2}$. The reason for this difference is, that the voltage difference between the readout strip and the accumulation layer increases with increasing oxide-charge density, and at the same time the width of the accumulation layer increases and extends below the metal 


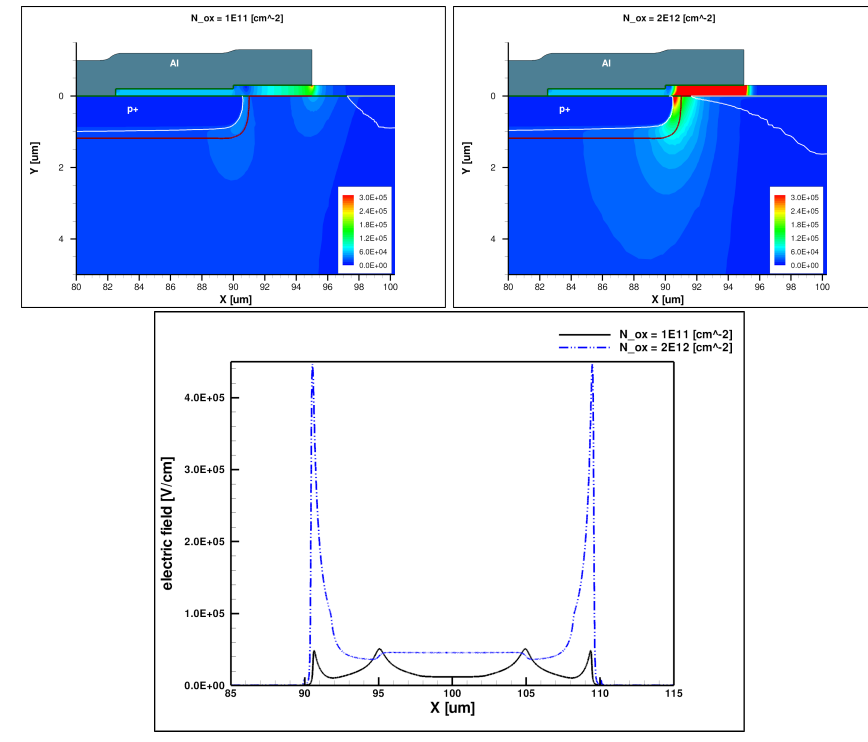

Figure 3: Influence of the oxide-charge density $N_{O x}$ on the electric field close ${ }^{170}$ to the $\mathrm{Si}_{-} \mathrm{SiO}_{2}$ interface. $2 \mathrm{D}$ simulation of a $p^{+}$strip sensor. Top left: $2 \mathrm{D}^{17}$ field distribution for $N_{o x}=10^{11} \mathrm{~cm}^{-2}$. Top right: $2 \mathrm{D}$ field distribution for 172 $N_{o x}=2 \times 10^{12} \mathrm{~cm}^{-2}$. Bottom: Electric field in the silicon $10 \mathrm{~nm}$ from the ${ }_{173}$ $\mathrm{Si}-\mathrm{SiO}_{2}$ interface for the two values of $N_{o x}$.

That X-ray radiation damage causes a significant reduction of the breakdown voltage, is also observed experimentally. Figure 4 shows for a sensor with a guard-ring structure consisting of one current-collection ring and 12 guard rings I-V curves for X-ray irradiations between 0 and $100 \mathrm{MGy}$. Whereas the non-irradiated sensor shows a "soft" breakdown around $900 \mathrm{~V}$, the breakdown voltage for an irradiated sensor can be as low as $250 \mathrm{~V}$. It should noted that this sensor has not been optimized for X-ray radiation hardness, and that the breakdown behavior in a dry ( $0.1 \%$ relative humidity) and a normal room atmosphere $(40 \%)$ is similar.

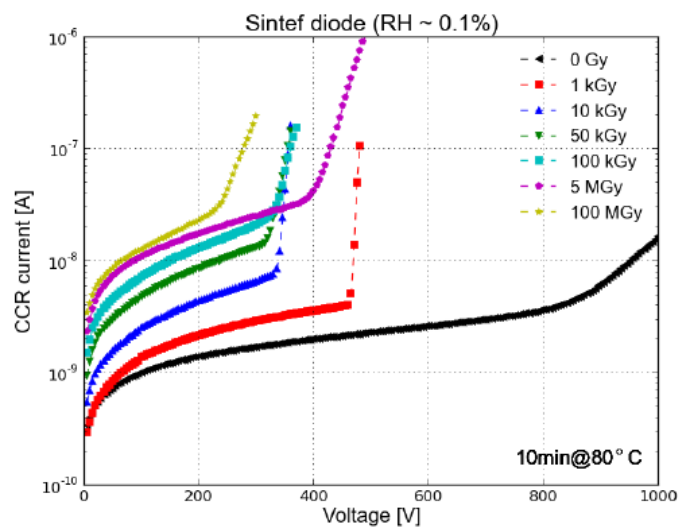

Figure 4: I-V characteristics of the Current Collection Ring of a Sintef sensor ${ }^{183}$ after annealing for 10 minutes at $80^{\circ} \mathrm{C}$ for different $\mathrm{X}$-ray doses.

The optimization of the AGIPD sensor has been performed ${ }_{186}$ with the help of 2D and 3D TCAD simulations [22]. We first present the optimization of the layout of the guard-rings and then of the pixels. For the effective oxide-charge density, values up to $3 \cdot 10^{12} \mathrm{~cm}^{-2}$, and for the current density, values up to $8 \mu \mathrm{A} / \mathrm{cm}^{2}$ have been assumed.

For the optimization of the guard-ring structure, we first made a 2D simulation of a single $p^{+}$strip on a $500 \mu \mathrm{m}$ thick $n$-type crystal of $5 \mathrm{k} \Omega \cdot \mathrm{cm}$ resistivity. The $p^{+}$strip, which was covered by aluminum overlapping the $\mathrm{SiO}_{2}$, was surrounded by a Current Collection Ring, CCR, and an $n^{+}$-scribe-line implant. For three values of oxide-charge densities $(1,2$, and $3 \cdot 10^{12} \mathrm{~cm}^{-2}$ ) the I-V characteristics was simulated for different depths of the $p^{+}$implant, dep, oxide thicknesses, $t_{o x}$, and aluminium overhang. The breakdown voltage $V_{b d}$ was obtained from the $\mathrm{I}-\mathrm{V}$ curve by the criterium $(\mathrm{d} I / \mathrm{d} V) /(I / V)=10$.

Figure 5 shows the results. For $N_{o x}=10^{12} \mathrm{~cm}^{-2}$ values for $V_{b d}$ above $200 \mathrm{~V}$ are found for $t_{o x}>300 \mathrm{~nm}$. For $3 \cdot 10^{12} \mathrm{~cm}^{-2}$ and $t_{o x} \gtrsim 300 \mathrm{~nm}, V_{b d}$ drops to about $20 \mathrm{~V}$. The reason is, that the silicon below the aluminium overhang depletes, and a highfield spike develops in the silicon at the corner of the $p^{+}$implant, as shown in Figure 3. From the simulations we conclude, that a breakdown voltage of $70 \mathrm{~V}$ can be reached for an oxide thickness of $270 \mathrm{~nm}$, a depth of the $p^{+}$implant of $2.4 \mu \mathrm{m}$, an aluminium overlap of $5 \mu \mathrm{m}$. We take these values as mpromise between high breakdown voltage and technological feasibility.

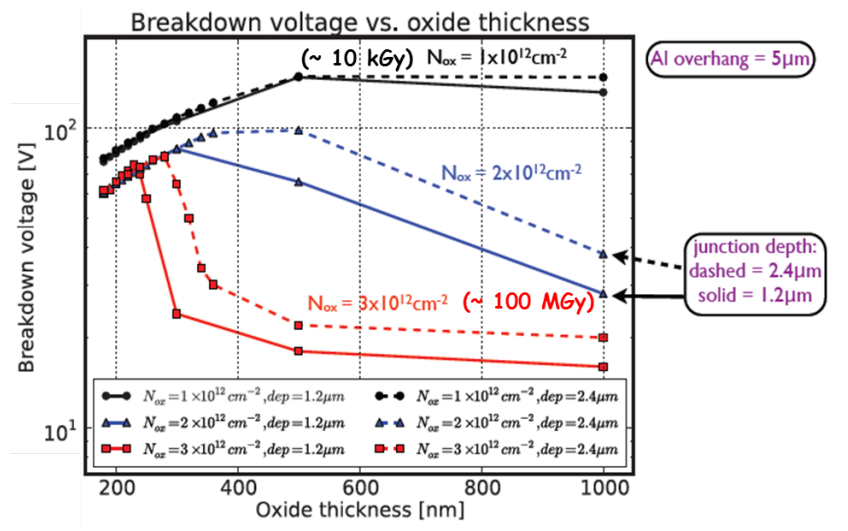

Figure 5: Breakdown voltage for a sensor surrounded by a CCR with zero GRs for different oxide charges, $N_{o x}$, two depths of the $p^{+}$implant, dep, as function oxide thickness.
With $V_{b d}=70 \mathrm{~V}$ without a guard ring, GR, we estimate that 15 GRs will be required to reach a breakdown voltage approaching $1000 \mathrm{~V}$. Next 2D simulations of a single strip and 15 GRs were performed. For $N_{o x}=3 \cdot 10^{12} \mathrm{~cm}^{-2}$ and the parameters obtained from the zero-GR optimization, the spacing between the GRs, their $p^{+}$-implant widths, and the aluminium age drop between adjacent GRs and minimum required space. Finally, a simulation in cylindrical coordinates of a circular pixel and GR layout was performed to verify that the breakdown voltage is not significantly smaller at the corners.

For low surface-charge densities, care has to be taken that the depletion region does not touch the scribe line, as this would overhang towards the strip have been optimized for equal volt- 
result in an excessive current in the CCR. To verify that this is 220 not the case, simulations were also made for $N_{o x}=5 \cdot 10^{10} \mathrm{~cm}^{-2}{ }_{221}$ as function of resistivity up to $12 \mathrm{k} \Omega \cdot \mathrm{cm}$.

In the optimization of the pixel layout, breakdown voltage, inter-pixel capacitance and dark current have been considered. To estimate the inter-pixel capacitance and the dark current, the ${ }^{223}$ values from $2 \mathrm{D}$ simulations have been extrapolated to the $3 \mathrm{D}_{224}$ situation using empirical formulae. Given that accumulation ${ }_{225}$ layers form at the $\mathrm{Si}_{-} \mathrm{SiO}_{2}$ interface, the inter-pixel capacitance ${ }_{226}$ depends only weakly on the distance between the $p^{+}$implants $_{227}$ of the pixels. As the dark current is given by $J_{\text {surf }} \cdot A_{d e p}$, where $A_{d e p}$ is the area of the depleted $\mathrm{Si}-\mathrm{SiO}_{2}$ interface, the aluminum 229 overhang and the gap between the $p^{+}$implants should be small..$_{230}^{29}$ A value of $20 \mu \mathrm{m}$ for the gap and $5 \mu \mathrm{m}$ for the overhang has ${ }_{23}$ been chosen.

Finally, a 3D simulation of a quarter of a pixel has been per- ${ }_{233}$ formed to verify the breakdown behavior and dark current. $\mathrm{As}_{234}$ symmetric boundary conditions were used, this corresponds to $_{235}$ the simulation of a complete pixel sensor. The simulations show ${ }_{236}$ that, with the optimized parameters, the specifications of the AGIPD sensor, in particular a breakdown voltage above $900 \mathrm{~V}$, a distance between the edges of the outer pixels and the cut edge ${ }^{237}$ of $1.2 \mathrm{~mm}$, an inter-pixel capacitance below $500 \mathrm{fF}$, and a dark ${ }_{238}$ current for the sensor of less than $50 \mu \mathrm{m}$ can be achieved for the ${ }_{239}$ values of $N_{o x}$ and $J_{\text {surf }}$, which correspond to X-ray dose values240 between 0 and 1 GGy. The optimized design of a corner of the $e^{241}$ sensor is shown in Figure 6.

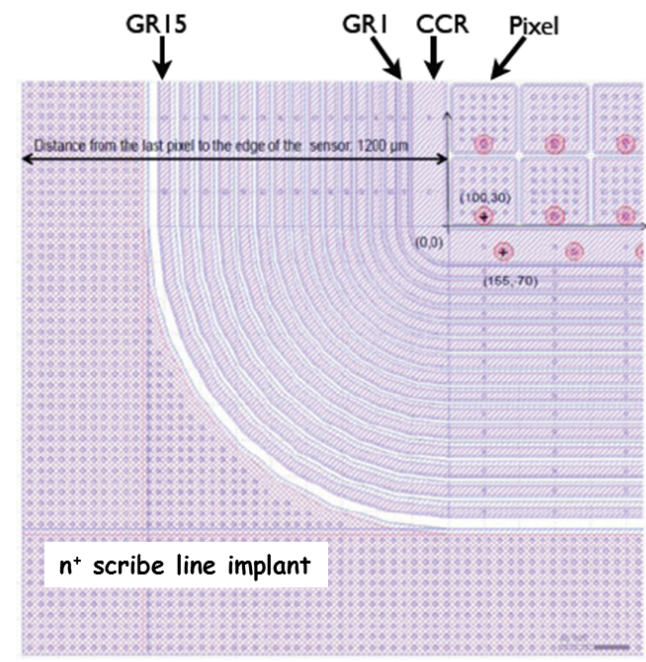

Figure 6: Layout of one corner of the AGIPD sensor. Starting from bottom left267 one sees the scribe lines, the $n^{+}$-scribe-line implant, the 15 guard rings, GR 15268 - GR 1, the Current Collection Ring, CCR and 6 pixels.

\section{Conclusions}

Experimental results from the study of radiation damage on ${ }_{276}^{275}$ test structures and sensors built on high-ohmic $n$-type silicon for $_{277}^{276}$ $\mathrm{X}$-ray doses in the range 0 to $1 \mathrm{GGy}$ have been presented. They 278 have been implemented in TCAD simulations and used for op-279 timizing the pixel sensor for the AGIPD (Adaptive Gain Inte- grating Pixel Detector) at the European X-Ray Free-Electron Laser. The simulations show that the specifications required for AGIPD can be met for X-ray dose values between 0 and 1 GGy.

\section{Acknowledgements}

R.K. thanks the organizers of the Vienna Conference of Instrumentation for the most pleasant, interesting, and exceedingly well organized conference. This work was performed within the AGIPD Project which is partially supported by the XFEL-Company. We would like to thank the AGIPD colleagues for the excellent collaboration. Support was also provided by the Helmholtz Alliance "Physics at the Terascale" and the German Ministry of Science, BMBF, through the Forschungsschwerpunkt "Particle Physics with the CMSExperiment". J. Zhang is supported by the Marie Curie Initial Training Network "MC-PAD", and I. Pintilie gratefully acknowledges the financial support from the Romanian Authority for Scientific Research through the Project PCE 72/5.10.2011.

\section{References}

[1] M. Altarelli et al. (Eds.), XFEL: The European X-Ray Free-Electron Laser, Technical Design Report, Preprint DESY 2006-097, DESY, Hamburg 2006, ISBN 978-3-935702-17-1, and http: //www . xfel .eu/de/.

[2] Th. Tschentscher et al., TECHNICAL NOTE XFEL.EU TN-2011-001 2011, DOI: 10.3204/XFEL.EU/TR-2011-001.

[3] H. Graafsma, 2009 JINST 4 P12011, DOI: $10.1088 / 1748$ 0221/4/12/P12011.

[4] R. Klanner et al., Challenges for Silicon Pixel Sensors at the European $X F E L$, to be published in Proceedings of RESMDD 13, subm. to Nucl. Instr. and Meth. A; arxiv:1212.5045.

[5] B. Henrich et al., Nucl. Instr. and Meth. A 663 (2011) S11-14, DOI: 10.1016/j.nima.2010.06.107.

[6] http://hasylab.desy.de/instrumentation/detectors/ projects/agipd/index_eng.html.

[7] P.A. Tove and W. Seibt, Nucl. Instr. and Meth. 51 (1967) 261

[8] J. Becker et al., Nucl. Instr. and Meth. A 615 (2010) 230-236, DOI: $10.1016 /$ j.nima.2010.01.082.

[9] J. Becker, Signal development in silicon sensors used for radiation detection, PhD thesis, Universität Hamburg, DESY-THESIS-2010-33 (2010).

[10] T. Poehlsen, et al., Nucl. Instr. and Meth. A 700 (2013) 22-39, DOI: $10.1016 / \mathrm{j} \cdot$ nima.2012.10.063.

[11] T. Poehlsen et al., Time dependence of charge losses at the $\mathrm{Si}_{-} \mathrm{SiO}_{2}$ interface in $p^{+} n$-silicon strip sensors, to be published in Proceedings of PIXEL2012, submitted to Nucl. Instr. and Meth. A.

[12] T.R Oldham, Ionizing Radiation effects in MOS Oxides, World Scientific Publishing Co. (1999).

[13] H.J Barnaby, IEEE Trans. Nucl. Sci. 53 (2006) 3103.

[14] Canberra Industries Inc., http://www.cismst.org.

[15] CiS Forschungsinstitut für Mikrosensorik und Photovoltaikk GmbH, http: //www. cismst.org.

[16] Hamamatsu Photonics, http://www.hamamatsu.com.

[17] Sintef ICT, http://www.sintef .no

[18] A.S. Grove, Physics and Technology of Semiconductor Devices, John Wiley \& Sons (1967).

[19] J. Zhang et al., 2012 JINST 7 C12012, DOI: 10.1088/1748$0221 / 7 / 12 / \mathrm{C} 12012$.

[20] J. Zhang et al., J. Synchrotron Rad. 19 (2012) 340-346, DOI: $10.1107 /$ S0909049512002348.

[21] E.H. Nicollian and J.R. Brews, MOS (Metal Oxide Semiconductor) Physics and Technology, New York, Wiley-Interscience, 1982.

[22] J. Schwandt, E. Fretwurst, R. Klanner, J. Zhang, 2013 JINST 8 C12015, DOI: $10.1088 / 1748-0221 / 8 / 01 / C 01015$. 